\title{
Continuous Hydrolysis and Liquid-Liquid Phase Separation of an Active Pharmaceutical Ingredient Intermediate Using a Miniscale Hydrophobic Membrane Separator
}

Cervera Padrell, Albert Emili; Morthensen, Sofie Thage; Lewandowski, Daniel Jacob; Skovby, Tommy; Kiil, Søren; Gernaey, Krist V.

\section{Published in:}

Organic Process Research and Development

Link to article, DOI:

10.1021/op200242s

Publication date:

2012

Document Version

Publisher's PDF, also known as Version of record

Link back to DTU Orbit

Citation (APA):

Cervera Padrell, A. E., Morthensen, S. T., Lewandowski, D. J., Skovby, T., Kiil, S., \& Gernaey, K. V. (2012).

Continuous Hydrolysis and Liquid-Liquid Phase Separation of an Active Pharmaceutical Ingredient Intermediate Using a Miniscale Hydrophobic Membrane Separator. Organic Process Research and Development, 16(5), 888900. https://doi.org/10.1021/op200242s

\section{General rights}

Copyright and moral rights for the publications made accessible in the public portal are retained by the authors and/or other copyright owners and it is a condition of accessing publications that users recognise and abide by the legal requirements associated with these rights.

- Users may download and print one copy of any publication from the public portal for the purpose of private study or research.

- You may not further distribute the material or use it for any profit-making activity or commercial gain

- You may freely distribute the URL identifying the publication in the public portal 


\title{
Continuous Hydrolysis and Liquid-Liquid Phase Separation of an Active Pharmaceutical Ingredient Intermediate Using a Miniscale Hydrophobic Membrane Separator
}

\author{
Albert E. Cervera-Padrell, ${ }^{\dagger}$ Sofie T. Morthensen, ${ }^{\dagger}$ Daniel J. Lewandowski, ${ }^{\dagger}$ Tommy Skovby, ${ }^{\ddagger}$ Søren Kiil, ${ }^{\dagger}$ \\ and Krist V. Gernaey* ${ }^{\dagger}$ \\ ${ }^{\dagger}$ Department of Chemical and Biochemical Engineering, Technical University of Denmark (DTU), Building 229, \\ DK-2800 Kgs. Lyngby, Denmark \\ ${ }^{\ddagger}$ Chemical Production Development, H. Lundbeck A/S, Oddenvej 182, DK-4500 Nykoebing Sj., Denmark
}

Supporting Information

\begin{abstract}
Continuous hydrolysis of an active pharmaceutical ingredient intermediate, and subsequent liquid-liquid (L-L) separation of the resulting organic and aqueous phases, have been achieved using a simple PTFE tube reactor connected to a miniscale hydrophobic membrane separator. An alkoxide product, obtained in continuous mode by a Grignard reaction in THF, reacted with acidic water to produce partially miscible organic and aqueous phases containing Mg salts. Despite the partial THFwater miscibility, the two phases could be separated at total flow rates up to $40 \mathrm{~mL} / \mathrm{min}$ at different flow ratios, using a PTFE membrane with $28 \mathrm{~cm}^{2}$ of active area. A less challenging separation of water and toluene was achieved at total flow rates as high as $80 \mathrm{~mL} / \mathrm{min}$, with potential to achieve even higher flow rates. The operability and flexibility of the membrane separator and a plate coalescer were compared experimentally as well as from a physical viewpoint. Surface tension-driven L-L separation was analyzed in general terms, critically evaluating different designs. It was shown that microporous membrane L-L separation can offer very large operating windows compared to other separation devices thanks to a high capillary pressure (Laplace pressure) combined with a large number of pores per unit area offering low pressure drop. The separation device can easily be operated by means of a back-pressure regulator ensuring flow-independent separation efficiency. Simple monitoring and control strategies as well as scaling-up/out approaches are proposed, concluding that membrane-based L-L separation may become a standard unit operation for continuous pharmaceutical manufacturing.
\end{abstract}

\section{INTRODUCTION}

Continuous separation of mixtures of immiscible liquids featuring different wettability properties can be achieved using microporous membranes. ${ }^{1-4}$ While in analytical chemistry this application has become a standard approach for sample enrichment and isolation, ${ }^{1-3}$ this function did not find its integration in the chemical engineering toolbox until recent years, with the emergence of microreaction technology. ${ }^{4,5}$ The aim of this relatively new field is the enhancement of phenomena such as mass transfer and heat transmission via large area to volume ratios, while taking advantage of surface tension effects at a small scale. ${ }^{5,6}$ These principles should be conserved across different scales of operation using a scaling-out approach, i.e. replicating small units working in parallel. ${ }^{6}$ However, in order to achieve common industrial acceptance, this technology must demonstrate factual scalability and robustness in operation under realistic (and thus challenging) scenarios. It is the purpose of this paper to demonstrate the applicability of a miniscale hydrophobic membrane-based separator for the continuous hydrolysis and liquid-liquid (L-L) separation of an organic and aqueous mixture, while critically evaluating the practical operability and limitations of micro-/miniscale-based L-L separators in the context of continuous pharmaceutical manufacturing (CPM).

The pharmaceutical industry is changing the way drug products are developed, manufactured, and distributed. ${ }^{7}$ A fierce competition between drug developers and generic manufacturers combined with an increasing cost for drug discovery and development is driving the industry to focus on efficient production, low manufacturing costs, and short delivery times. ${ }^{7}$ Continuous pharmaceutical manufacturing (CPM), compared to batch production, has shown some advantages in terms of mass and heat transfer, volume of potentially flammable or toxic substances, accessibility to extreme working conditions in terms of pressure and temperature, and other advantages, ${ }^{8-10}$ while being most suitable for the application of the concepts of quality by design $(\mathrm{QbD})^{11}$ and process analytical technology (PAT) ${ }^{12}$ In this context, microreaction technology and microbased separations ( $\mu \mathrm{m}$ scale) have rapidly developed in recent years, ${ }^{5}$ providing the basic tools to achieve multistep organic synthesis in continuous mode. ${ }^{13,14}$ The miniaturization of reaction and separation devices is especially advantageous in process development studies and high-throughput screening (where reagents and catalysts may be scarce, harmful and/or expensive), motivating the expansion of microfluidic devices and techniques. In contrast, in our opinion, mini-/mesoscale reaction and separation technology $(\mathrm{mm}-\mathrm{cm}$ scale) have received relatively less attention, even though it is potentially possible to access some of the advantages

Special Issue: Continuous Processes 2012

Received: August 31, 2011

Published: February 21, 2012 
of small scales while lowering the sensitivity to practical problems such as blockages from solids in suspension, which still form a main limitation for microreaction technology. ${ }^{5,15}$ Furthermore, it has been observed that fluid distribution and parallelization of microscale units is not such a straightforward task, despite expectations based on the standard scaling-out principles. ${ }^{16}$ Thus, constructing basic units at a moderately larger scale should theoretically make scaling-up/-out an easier and smoother task.

$\mathrm{L}-\mathrm{L}$ extraction and separation is one of the basic unit operations with the widest application in the chemical industry and in particular in the pharmaceutical industry. At macroscale, countercurrent flow is accomplished by gravitational segregation involving droplets, ${ }^{17}$ whereas in batch mode, L-L extraction and separation are usually achieved by simple mixing and decantation operations, the latter being driven by density differences. However, when density differences are small, the use of simple decantation may lead to prohibitively long settling times. The use of hydrocyclones may partially solve this problem. For example, the centrifugal contactor separator (CCS) has found applications in biodiesel production $^{18,19}$ and for the enantioselective separation of amino acids, ${ }^{20,21}$ among others, due to its attractive integration of different functions (mixing-reaction-separation) in one unit, ${ }^{19}$ as well as the possibility to connect several units in a cascade system. ${ }^{20,21}$ However, while this technology has found acceptance in industry, it is difficult to implement at laboratory scale, since the smallest units available require relatively high volumes of potentially expensive and/or hazardous materials. Thus, development of such units must start directly in pilot scale. ${ }^{18-21}$

At the micro- and miniscales, when the Bond number (eq 1, where $\rho$ is the density, $g$ is the gravity acceleration, $L$ is a characteristic length, and $\gamma$ is the surface tension) is less than 1 , surface tension becomes more important than gravity, and thus the most efficient driving force for separation. ${ }^{4,5} \mathrm{~L}-\mathrm{L}$ extraction involves two main operations, namely mixing or interfacial contact (where extraction occurs) and phase separation, which may be integrated in a single unit or divided into several ones. As reviewed by Hartman and Jensen, ${ }^{5}$ cocurrent and countercurrent arrangements for interfacial contact have been achieved using segmented (cocurrent) flow or laminated (co- or countercurrent) flow, the latter requiring hydrophobic and hydrophilic walls to focus the two immiscible phases. In addition, it should also be possible to use microporous membrane liquid-liquid extraction (MMLLE) and related techniques as employed in analytical chemistry. ${ }^{2,3}$ While countercurrent laminated flow is expected to provide a theoretical plate number larger than 1 (e.g., up to 4.6 as demonstrated by Aota et al. ${ }^{17}$ ), this may be feasible only at very low flow rates, possibly due to mixing limitations requiring a long residence time. Okubo et al. ${ }^{22}$ compared the mass transfer rates obtained with three different extraction operations, i.e. contact flow in a Y-shaped microchannel (laminated flow), segmented flow and emulsification. They concluded that slow molecular diffusion in a contact flow microchannel limits its applicability, while segmented flow and emulsification provided more efficient mixing. ${ }^{22}$ Segmented flow showed a lower mixing rate compared to emulsification, however the two immiscible phases were more readily separated than the emulsions obtained with a standard micromixer. $^{22}$ It has actually been proposed to use plate coalescers in order to reduce the degree of emulsification prior to actual separation. ${ }^{22-25}$

$$
B_{\mathrm{o}}=\frac{\rho g L^{2}}{\gamma}
$$

Subsequent separation of the immiscible phases may be performed by flow focusing, ${ }^{26}$ manipulation of surface wetting characteristics, ${ }^{16,17,24,27,28}$ or using capillary forces through a microporous hydrophobic membrane. ${ }^{1,3,4}$ Another recent trend is to use hydrophobic ducts ${ }^{29}$ through which an organic phase may be separated (somewhat similar to a hydrophobic membrane, but with larger pores and in fewer number), or to stabilize the interface of two contacting phases with the use of micropillars. ${ }^{30}$ These two last approaches avoid the use of a hydrophobic membrane, and as reported by Castell et al., ${ }^{29}$ small solids in suspension $(2 \mu \mathrm{m} \text { size })^{29}$ can be handled more easily.

It can be concluded that, in contrast to the widely used CCS in pilot scale, a large number of studies have been published demonstrating the use of L-L extraction and separation at microscale, with the largest flow rates reported in the order of 1-2 $\mathrm{mL} / \mathrm{min}^{4,29}$ However, no work has been found demonstrating or discussing the scale-up/-out of these devices and their flexibility for practical use. Note also that many of these reports are experimental, with only some of them performing a physical analysis of the system. ${ }^{4,16,26,28-31}$ Since one of the claimed advantages of continuous processing and microreaction technology is a smooth transition between scales of operation, it is critical to demonstrate that laboratory equipment provides reliable representations of full-scale equipment.

The objective of this paper is thus to analyze the requirements for successful surface tension based phase separation, following the rules introduced by Aota et al. ${ }^{31,32}$ and Kralj et $\mathrm{al}^{4}$ and which have been presented elsewhere in similar formulations. Subsequently the suitability and flexibility of different L-L extraction/separation technologies will be assessed for large-scale industrial operation as part of continuous pharmaceutical production processes. Two experimental setups were constructed: a plate coalescer similar to the one proposed by Kohlemainen and Turunen ${ }^{24}$ and a miniscale PTFE membrane separator based on the concepts developed by Kralj et al. ${ }^{4}$ The two setups were preliminarily tested with a mixture of water and toluene to confirm practical operability and flexibility, with the PTFE membrane separator achieving a flow rate up to $40 \mathrm{~mL} / \mathrm{min}$ per phase with perfect separation. This flow rate is in the same order of magnitude as the one expected for industrial-scale continuous pharmaceutical production (e.g., Barthe et al. $^{33}$ and Roberge et al. ${ }^{34}$ report an industrial-scale flow rate of $80-100 \mathrm{~g} / \mathrm{min}$ for a continuous Grignard reaction typical for continuous API synthesis).

Once the operability of different surface-tension based L-L separation approaches was physically analyzed and experimentally validated with the simple water-toluene separation, a more challenging reaction and separation system was tested. The product of a Grignard alkylation reaction obtained in continuous mode was hydrolyzed in a tube reactor and the organic and aqueous phases resulting from this hydrolysis step were subsequently separated using the aforementioned PTFE membrane separator. The Grignard reaction is one of the most useful synthetic building blocks for the pharmaceutical industry. ${ }^{35,36}$ This very fast and exothermic reaction ${ }^{37}$ has previously been carried out in continuous mode under homogeneous conditions in microreactors, ${ }^{35}$ optionally feeding the Grignard reagent through multiple injections or sideentries. $^{33,34,38}$ The reactor system employed in this work, developed by Müller et al., ${ }^{39}$ combines a heterogeneous phase filter reactor ${ }^{39}$ with a homogeneous phase side-entry reactor ${ }^{38}$ in order to achieve high product concentrations with low solvent consumption. ${ }^{39}$ After hydrolysis, two phases are obtained: an 
organic phase consisting of THF dissolving an active pharmaceutical ingredient (API) intermediate at high concentrations $(\sim 200-500 \mathrm{~g} / \mathrm{L})$, and an aqueous phase containing $\mathrm{Mg}$ salts. Due to the partial THF-water miscibility, this phase separation relying on surface tension is especially challenging. Despite the high API intermediate concentration, the organic phase could be separated with total recovery of the API intermediate, without experiencing fouling or clogging problems in the short term studies carried out. This application thus demonstrates the potential of hydrophobic membrane based separation for industrially relevant and challenging solutions at close to industrial-scale flow rates. The active membrane area was only $28 \mathrm{~cm}^{2}$, and therefore, flow rate improvements can be expected, following a scaling-up approach, with only a few or no replicate parallel units. Since the Grignard product was produced using a continuous flow reactor, multistep organic synthesis combining reaction plus subsequent L-L separation was demonstrated at miniscale (tens of $\mathrm{mL}$ range). To our knowledge, this flow rate range has only previously been achieved using hydrocyclones combining reaction-extraction-separation, meaning that surface tension-based L-L separation and enhanced gravity-based separation can potentially compete at medium flow rates.

\section{PHYSICAL CONSIDERATIONS DRIVING THE DESIGN}

2.1. Flow-Dependent Pressure Gradient vs Laplace Pressure. Successful L-L phase separation at the micro-/ miniscale (Bond number $<1$ ) depends on the balance of a flowdependent pressure gradient built between the two phases, and the Laplace pressure stabilizing the interface. The pressure drop of a liquid flowing in a round channel or a tube in the laminar regime may be calculated using eq 2 , where $\mu$ is the viscosity, $Q$ is the flow rate, $L$ is the length, and $R$ is the radius. ${ }^{4}$ For rectangular channels of width $w$ and height $h$, the pressure drop may be approximated by eq 3 , given that $h<w$, with an error less than $13 \%$, even in the worst case when $h=w^{40}$ Thus, the pressure drop depends on the dimensions of the channel, the flow rate, and the viscosity. Assuming that two different phases are successfully separated into two different areas of a channel or alternatively into two or more different channels connected in some manner, differences in any of these parameters may cause a significant difference in pressure drop between the two phases. Furthermore, if fluidic connections are attached to the separation device, the pressure gradient in the system will also depend on the pressure drop caused by the flow in these connections plus any source of downstream backpressure. Therefore in general, the pressure for any phase at the inlet of the separation device is given by the sum of the pressure drop in the channel, the pressure drop in the fluidic connections, and the downstream back-pressure $^{31,32}$ (eq 4.). A flow-dependent pressure gradient between the two phases is thereby generated ${ }^{32}$ (eq 5).

$$
\begin{aligned}
& \Delta P_{\text {tube }}=\frac{8 \mu Q L}{\pi R^{4}} \\
& \Delta P_{\text {rec.channel }} \approx \frac{12 \mu Q L}{h^{3} w}\left(1-0.63 \frac{h}{w}\right)^{-1} \\
& \Delta P^{\text {phase } i}=\Delta P_{\text {channel }}+\Delta P_{\text {tube }}+\Delta P_{\text {back-pressure }} \\
& \Delta P^{\text {flow }}=\Delta P^{\text {phase } 1}-\Delta P^{\text {phase } 2}
\end{aligned}
$$

The pressure gradient between the two phases must be stabilized by the Laplace pressure at the interface, which in a channel as in Figure 1 lies between the values given by the

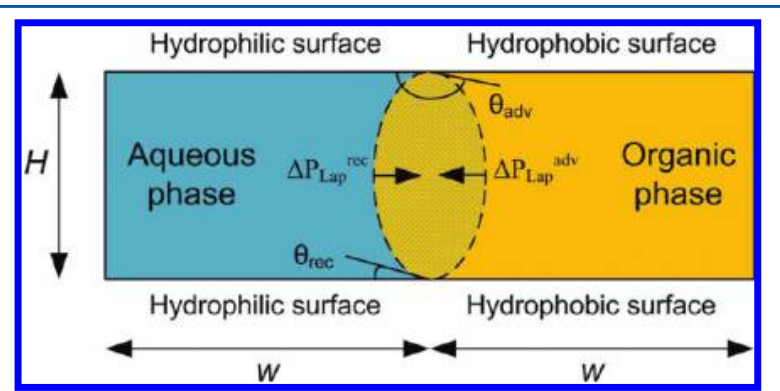

Figure 1. Representation of the Laplace pressure given by the advancing and receding contact angles for the particular case where the interface is lying between a hydrophilic and a hydrophobic surface. The direction of the flow could for example be perpendicular to the plane, similar to the devices reported by Aota et al. ${ }^{17,31,32}$.

advancing and receding contact angles as shown by eq 6 , where $\gamma$ is the surface tension between the two fluids and $\theta_{\text {adv }}$ and $\theta_{\text {rec }}$ are the advancing and receding contact angles, respectively. ${ }^{31,32}$ The interface may be easier stabilized when it lies between two solid surfaces with different hydrophilicity. ${ }^{32}$ Figure 1 shows an example of a theoretical separation device, where a rectangular channel of width $2 w$, height $h$, and length $L$ will be used to separate an aqueous phase and an organic phase in two areas, each with a width $w$, which are treated individually for calculation of the pressure drop. The left and right sides of the channel have hydrophilic and hydrophobic surfaces, respectively. Assuming that the aqueous phase will lie in the hydrophilic area, if it is pushed to the hydrophobic area it will receive a Laplace pressure in the opposite direction given by the advancing contact angle between the aqueous phase on the hydrophobic surface surrounded by organic phase (Note that the advancing contact angle $\theta_{\text {adv }}$ is larger than $90^{\circ}$, and thus the $\cos \theta_{\text {adv }}$ is negative). If, on the contrary, the organic phase is pushed to the hydrophilic area, it will receive a Laplace pressure in the opposite direction given by the receding contact angle between the aqueous phase on the hydrophilic surface surrounded by organic phase $\left(\theta_{\text {rec }}\right)$.

$$
\frac{2 \gamma \cos \theta_{\mathrm{adv}}}{h}<\Delta P^{\text {Laplace }}<\frac{2 \gamma \cos \theta_{\mathrm{rec}}}{h}
$$

2.2. Conditions for Phase Separation. The two conditions for a successful phase separation can be stated as in eq 7.1 and $7.2 .^{31,32}$ If any of the two conditions are not fulfilled, either the aqueous phase will leak on the hydrophobic side $\left(\Delta P^{\text {flow }}>\Delta P_{\text {adv }}^{\text {Lap }}\right)$, or the organic phase will leak on the hydrophilic side $\left(\Delta P^{\text {flow }}>\Delta P_{\text {rec }}^{\text {Lap }}\right){ }^{31}$ From eqs 4,5 , and 7 it is possible to determine the flow parameters which will lead to a successful separation for a given mixture. This would for example involve assuming a certain flow rate of the mixture and a given flow ratio of the organic and aqueous phases to determine the right dimensions of the separation channels and those of the fluidic connections. Another application could be determining the flow ratio of an organic and an aqueous mixture which, for a desired total flow rate, could be successfully separated in a given device. ${ }^{17}$ However this approach is basically limited to demonstration purposes, since the operating window for a separator device will be very much constrained to the original design parameters. Furthermore, any disturbances in the 
system would be very difficult to reject thus causing leakage of any of the two phases on the wrong channels.

$$
\Delta P^{\text {flow }}<\Delta P_{\text {adv }}^{\mathrm{Lap}}(7.1) \text { and } \Delta P^{\text {flow }}>\Delta P_{\text {rec }}^{\text {Lap }}(7.2)
$$

A first attempt to solve this problem could be installing a feedback pressure controller using a pair of back pressure regulators in the two outlets of the separation device. The controller would ensure that the pressure gradient between the two outlets fulfills eq 7. Yet, it would not be possible to control the flow-dependent pressure gradient throughout the device. It is thus possible that eq 7 is fulfilled at the outlet of the separator but not in the inlet, potentially impairing its performance. As an example, consider a separator for which the cross-sectional area is as in Figure 1, and the length is $5 \mathrm{~cm}$. A flow $Q$ is introduced in the device, containing 50\% water and 50\% toluene. Since water is more viscous than toluene, its pressure drop will be larger and thus a pressure gradient will be built, pushing the water towards the hydrophobic area. The Laplace pressure at the interface will partially compensate for this as long as eq 7.1 is respected. Figure 2 shows the Laplace pressure given by the

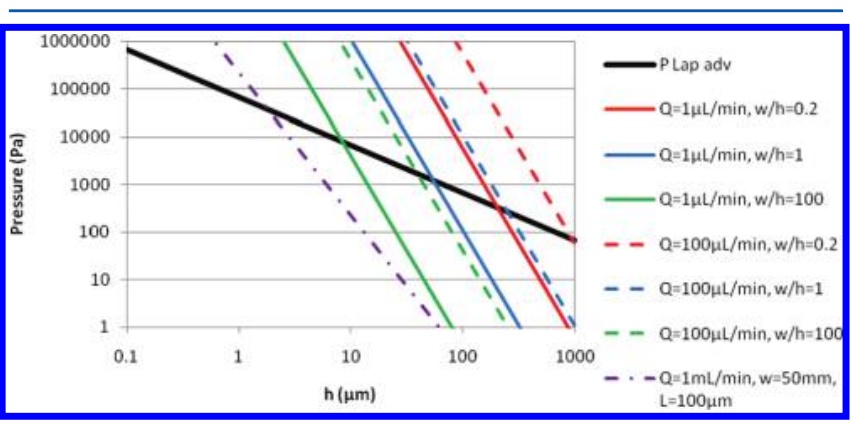

Figure 2. Laplace pressure assuming an advancing contact angle of $160^{\circ}$ and a surface tension as for water-toluene $(36 \mathrm{mN} / \mathrm{m})$, for different values of the channel height (black continuous line); flowdependent pressure gradient for different aspect ratios $w / h$, two different flow rates $Q$ and fixed value of length $(5 \mathrm{~cm})$ (red, blue and green lines); pressure drop caused by toluene in a $50 \mathrm{~mm}$ wide, $100 \mu \mathrm{m}$ long and height $h$ connection channel between two $1 \mathrm{~mm}$ diameter flow channels as shown in Figure 3, at a flow rate of $1 \mathrm{~mL} / \mathrm{min}$ (purple line).

advancing contact angle of water (eq 6), assuming it is a very hydrophobic surface $\left(\theta_{\mathrm{adv}}=160^{\circ}\right)$, using the surface tension for water-toluene $(36 \mathrm{mN} / \mathrm{m})$ for different values of the channel height $h$. The figure also shows the flow-dependent pressure gradient for different aspect ratios $w / h$ and two different flow rates $Q(1 \mu \mathrm{L} / \mathrm{min}$ and $100 \mu \mathrm{L} / \mathrm{min})$, using eq 3 . The operating window is thus the area where the flow-dependent pressure gradient is lower than the Laplace pressure. It can be observed that the Laplace pressure is very low (values given in $\mathrm{Pa}$ ) except for very small channel heights (e.g., $7 \mathrm{kPa}$ and above only below $10 \mu \mathrm{m})$. Since it would be desired to have a simple pressure controller to regulate the separation, it would be necessary to use a small channel height. However, channels with small height entail a high pressure gradient, thus disrupting the interface.

2.3. Decoupling the Flow-Dependent Pressure Gradient and the Laplace Pressure. One way to decouple the flow-dependent pressure gradient and the Laplace pressure at the interface is to design a separator with large flow channels and a small interface, for example using a cross-sectional area as shown in Figure 3. The diameter of the channels has been chosen as $1 \mathrm{~mm}$ (Bo number $=0.27$ ), the length of the device is

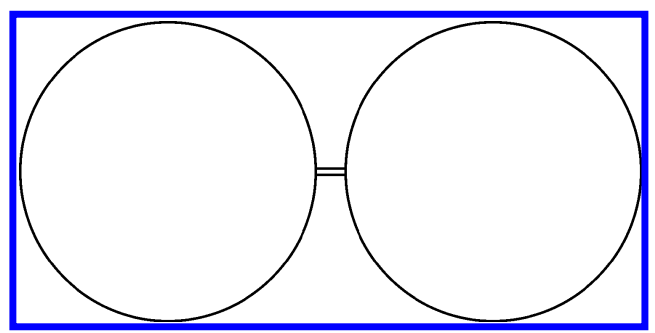

Figure 3. Schematic cross-sectional area of a conceptual separation device where two $1 \mathrm{~mm}$ fluidic channels for aqueous phase (left) and organic phase (right) are connected by a $100 \mu \mathrm{m}$ long and $10 \mu \mathrm{m}$ high rectangular channel. The mixture enters the left side, and the organic phase has to "squeeze" through the connection channel to the right side.

$5 \mathrm{~cm}$ and the interface is a rectangular channel of length $100 \mu \mathrm{m}$ and height $10 \mu \mathrm{m}$ (and width $5 \mathrm{~cm}$ ). If the inlet is in the left channel, the organic phase will need to squeeze through the interface to reach the right channel. This could be achieved by applying a back pressure at the outlet of the aqueous phase lower than the Laplace pressure (so that water does not leak into the right channel), but high enough to exceed the pressure drop in the connection channel. The pressure drop caused by toluene in the connection channel has been plotted in Figure 2. For a value $h=10 \mu \mathrm{m}$, it can be observed that while the Laplace pressure is $7 \mathrm{kPa}$, the pressure drop to cross the connection channel is $0.2 \mathrm{kPa}$ for a flow rate of $1 \mathrm{~mL} / \mathrm{min}$, and it could be lowered by increasing the length of the device, since the pressure drop for water in the left channel is only $0.03 \mathrm{kPa}$. Another option would be decreasing the length of the connection channel below $100 \mu \mathrm{m}$, or increasing the number of connection channels. This is an example of the conclusions reached in some previous works. ${ }^{29,30}$

Yet, one can note that in order to achieve significant Laplace pressures (in the order of 0.1 to $1 \mathrm{bar}$ ), it is required to use very narrow interfaces, and this causes very rapidly an increase in the pressure drop at the interface. In practice, however, this can very easily be done using a hydrophobic membrane separating two large channels, ${ }^{4}$ since the connecting channels have a very small diameter (porosity from 0.1 to $1 \mu \mathrm{m}$ readily available) but also the membranes contain a large number of them (in the order of $10^{7}-10^{8}$ pores $/ \mathrm{cm}^{2}$ ) and are very thin (typically below $100 \mu \mathrm{m}$ ). In this way, a membrane based separator can be built by calculating the required area (the required number of pores $n$ ) for a desired range of operating flow rates using eq $8 .^{4}$

$$
\Delta P_{\text {membrane }}=\frac{8 \mu Q L}{n \pi R^{4}}
$$

\section{MATERIALS AND METHODS}

3.1. Construction of a Plate Coalescer. Two prototypes of a plate coalescer similar to the one proposed by Kolehmainen and Turunen $^{24}$ were built. A plate coalescer consists of a flat rectangular microchannel limited by a hydrophobic surface on one side and a hydrophilic surface on the opposite side. The first prototype was built using a $3 \mathrm{~cm}$ wide, $2 \mathrm{~cm}$ high and $24 \mathrm{~cm}$ long PTFE block. On one of the $3 \times 24 \mathrm{~cm}$ surfaces a $100 \mu \mathrm{m}$ deep, $2 \mathrm{~cm}$ wide and $20 \mathrm{~cm}$ long microchannel was milled using a CNC machine. This surface was covered by a glass plate (hydrophilic surface), pressed against the PTFE block using some clamps. On the PTFE block two $1 / 8$ in. $(3.2 \mathrm{~mm})$ holes were drilled for the fluidic connections (inlet and outlet). The glass plate had a $2 \mathrm{~mm}$ hole 
with a $4 \mathrm{~mm}$ OD glass tube for the aqueous outlet. It was found very difficult to avoid leakages in this system, and in the end the glass plate broke due to unevenly distributed pressure from the clamps. However, some preliminary tests were done which allowed observing the separation phenomena (if any) through the transparent glass.

The second prototype was much more robust in construction. It consisted of two stainless steel plates, one with a low hydrophobic surface (stainless steel) and one with a hydrophobic surface. ${ }^{24}$ A $1 \mathrm{~mm}$ thick fluoro-polymer-based coating (Accotron, Accoat A/S) was indeed applied to one of the stainless steel plates, in order to create the hydrophobic surface. On the bare stainless steel plate, a flat rectangular microchannel was machined in a similar way and with similar dimensions as for the first prototype (channel length around $10 \mathrm{~cm}$ ). However this time leakages were completely avoided by machining an outer groove where a flexible O-ring was inserted. When the two plates were pressed against each other using 10 fixing screws, the O-ring was compressed and could thereby avoid any leakages which could be caused by tiny irregularities on the stainless steel surface. In the organic-phase outlet a $1 / 8 \mathrm{in}$. $(3.2 \mathrm{~mm})$ O.D. PFA tube from Swagelok was inserted. The tube reached the Accotron surface and was kept in place using 1/4-28 Upchurch connections without leakages. In the aqueous phase outlet a $1 / 8$ in. Swagelok male union was welded, to which a $1 / 8$ in. stainless steel tube was attached. The two outlets (PFA tube and stainless steel tube) were connected to two check valves with a cracking pressure adjusted to 8 psi and subsequently to 2 PTFE back-pressure regulators (1/4 in. Partek, $0-30$ psig backpressure, Parker) (Figure 4c). It was otherwise found to be very

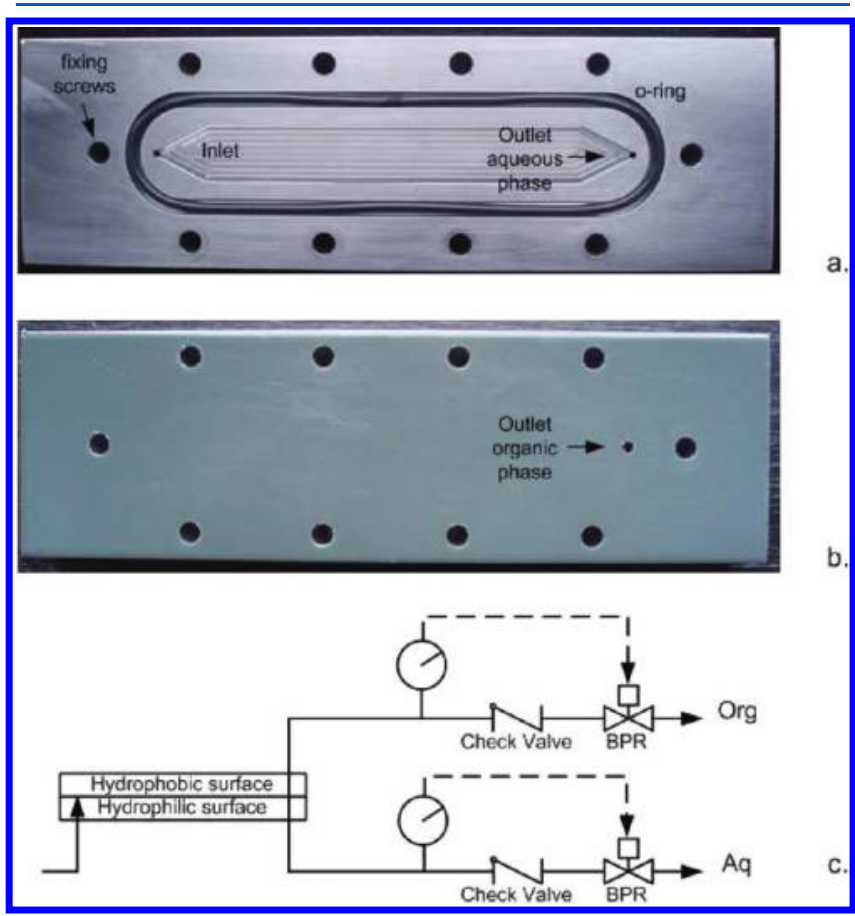

Figure 4. (a) Stainless steel plate (low hydrophobic surface). (b) Accotron-coated stainless steel plate (hydrophobic surface). (c) Scheme of the separation setup. (BPR = back-pressure regulator).

difficult to control the pressure drop in these outlets, since small changes in height, flow rate, or length of the tubes would cause differences in pressure drop larger than the Laplace pressure given by the separator device. The check valves were useful to avoid any backflow caused by a wrong regulation of any of the two back-pressure regulators.

3.2. Construction of a PTFE Membrane Separator. A PTFE membrane separator was built using 2 stainless steel discs, between which a $10 \mathrm{~cm}$ diameter PTFE membrane (Sartorius Stedim) could be sandwiched. Two different pore sizes were tested: $0.2 \mu \mathrm{m}$ with $65 \mu \mathrm{m}$ thickness and $0.45 \mu \mathrm{m}$ with $80 \mu \mathrm{m}$ thickness. The discs were machined by milling to obtain a $1 \mathrm{~mm}$ deep, $9.5 \mathrm{~mm}$ wide rectangular channel on the first disk (Figure 5a, aqueous side) and a $2 \mathrm{~mm}$ deep, $9.5 \mathrm{~mm}$ wide rectangular channel on the second disk (Figure $5 \mathrm{~b}$, organic side). The total length of the channels was a little above $22.5 \mathrm{~cm}$ (without considering the turns). The contact area between the channels and the membrane was $28 \mathrm{~cm}^{2}$. On the second disk, a $60 \mu \mathrm{m}$ deep circular groove was machined around the channel in order to place a mesh which could be used to support the PTFE membrane. Otherwise there could be the risk that the PTFE membrane could bend too much towards the organic side (note the pressure gradient through the membrane), thus blocking the organic side channel or simply breaking. However, it was found that the mesh was damaging the membrane by compression and it was actually not necessary, since the PTFE membranes were strong and flexible enough for this operation. The first disk had two round grooves around the channel used to insert 2 flexible o-rings. The inner O-ring was planned to be used to press the PTFE membrane against the supporting mesh (later found unnecessary). The second O-ring was used to compress the PTFE membrane against the stainless steel surface and avoid any leakages. In the end, after removing the mesh, the two o-rings just served the function of avoiding leakages and showed very reliable. Eight screws were used to fix the two discs together, and two pins indicated the right positioning of the discs.

The fluidic connections of the membrane separator were done by means of 1/4-28 Upchurch connections. 1/8 in. $(3.2 \mathrm{~mm})$ PFA tubes from Swagelok were used, and stainless steel and PFA Swagelok connectors were used to connect the aqueous side outlet to a manometer and a PFA back pressure regulator (1/4 in. Partek, 0-30 psig back pressure, Parker) (Figure 5c). The apparent inlet of the organic side was used for washing the separator and could potentially be used for reversing the flow of the separator to remove any fouling or blockages on the opposite side.

3.3. Preliminary Experiments: Separation of Water and Toluene. Distilled water colored with methylene blue and toluene from Sigma Aldrich were used for the preliminary separation experiments. Preliminary experiments were carried out using the plate coalescer and the PTFE membrane separator, testing different flow rates and flow ratios of water and toluene. Since the water was colored, it was easy to evaluate when the separation was successful by a simple observation of the outlet streams. The minimum and maximum back pressures giving a successful separation were noted for each experiment.

3.4. Continuous Hydrolysis of an Alkoxide Product from a Grignard Reaction. Allylcarbinol (3, Scheme 1) is the short name for 9-Allyl-2-Chlorothioxanthen-9-Ol, an intermediate product in the production of zuclopenthixol, an API developed by $\mathrm{H}$. Lundbeck A/S. It is produced via an alkylation reaction (Grignard reaction) of 2-chlorothioxantone (1, short name CTX), as shown in Scheme 1. The product of this reaction is an alkoxide (2, Scheme 1$)$. Without the addition of acid to the hydrolysis, the reaction product would form insoluble basic $\mathrm{Mg}$ salts of the form $\mathrm{MgCl}(\mathrm{OH})$ or $\mathrm{Mg}(\mathrm{OH})_{2}$. With the addition of 

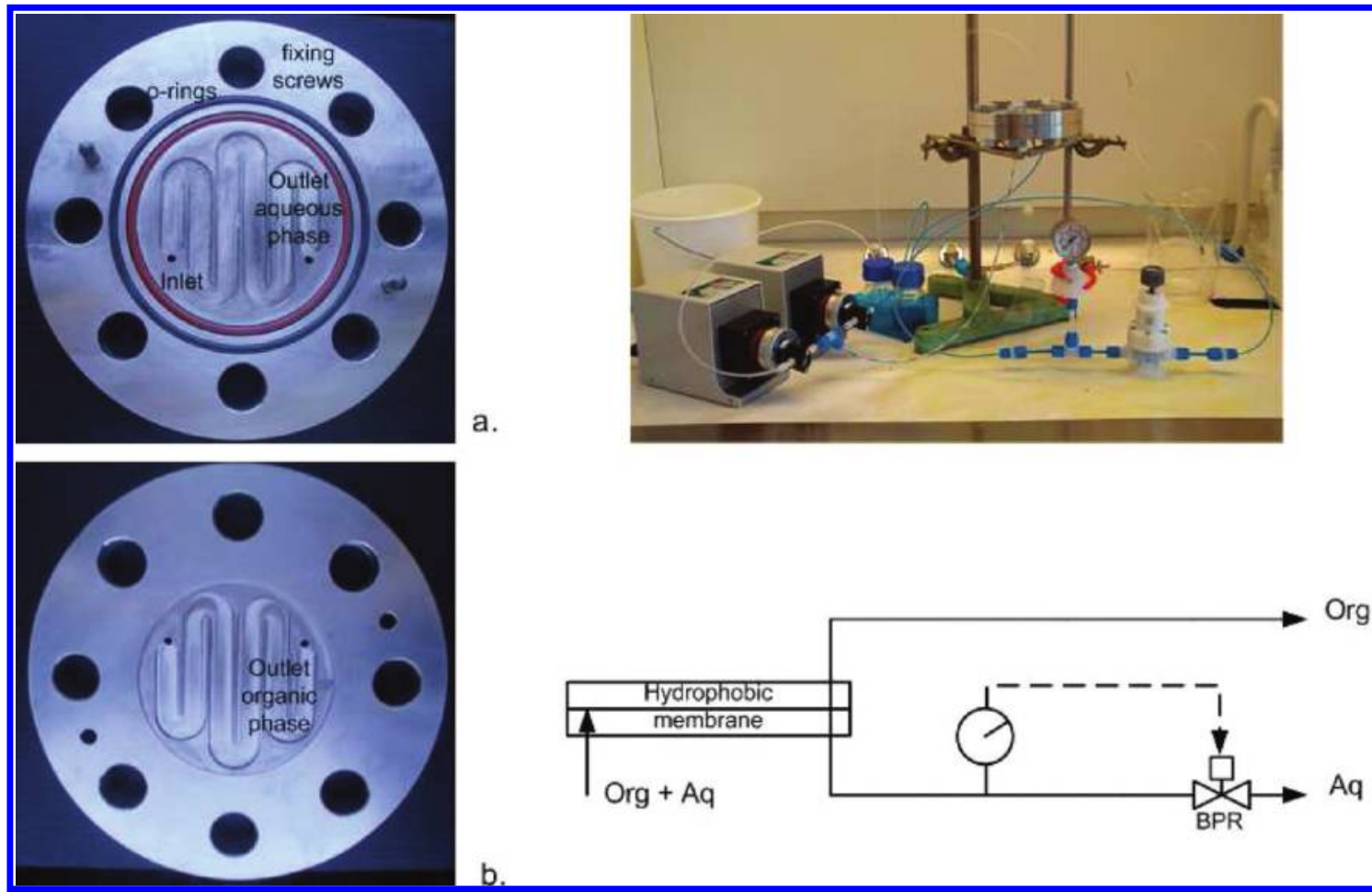

Figure 5. (a) Stainless steel $1 \mathrm{~mm}$ channel for the aqueous phase, (b) stainless steel $2 \mathrm{~mm}$ channel for the organic phase, (c) picture of the membrane separator setup, where the blue solution is water and the uncolored liquid is toluene, (d) scheme of the separation setup.

Scheme 1. Alkylation and hydrolysis reaction

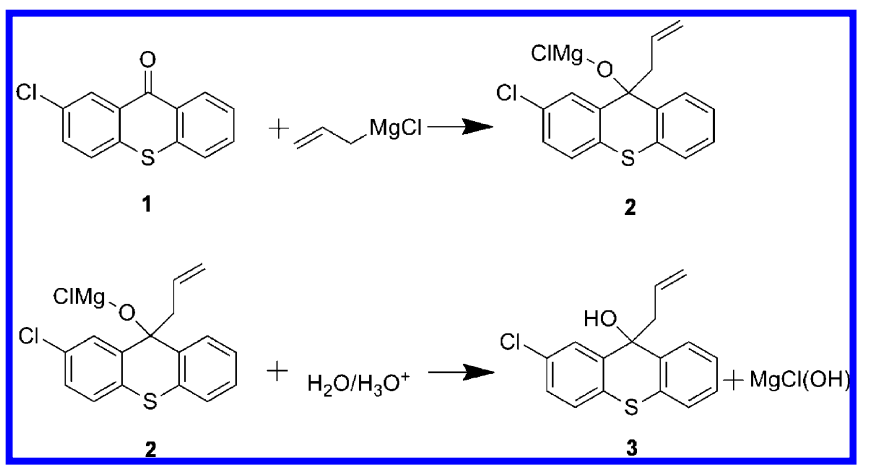

$\mathrm{HCl}$ to the water used for hydrolysis, these $\mathrm{Mg}$ salts are partially or totally solubilized in the form of $\mathrm{MgCl}_{2}$. Two partially insoluble phases are formed, an organic phase containing THF, a small amount of water, allylcarbinol and other organic impurities, and an aqueous phase containing water, THF and partially soluble $\mathrm{MgCl}_{2}$ salts. THF and water are totally miscible unless the $\mathrm{MgCl}_{2}$ salts and allylcarbinol are present (salting-out effect $^{41}$ ), in which case they are only partially miscible.

3.4.1. Continuous Grignard Alkylation Reactor. The alkylation reaction (Scheme 1, first line) was carried out in a filter reactor $^{39}$ coupled with a side-entry reactor ${ }^{38}$ as shown in Figure 6. The filter reactor was a $1 \mathrm{~L}$ stirred vessel (Ace Glass Inc.) operated in fed-batch mode, since only about $1 \mathrm{~L}$ of product was

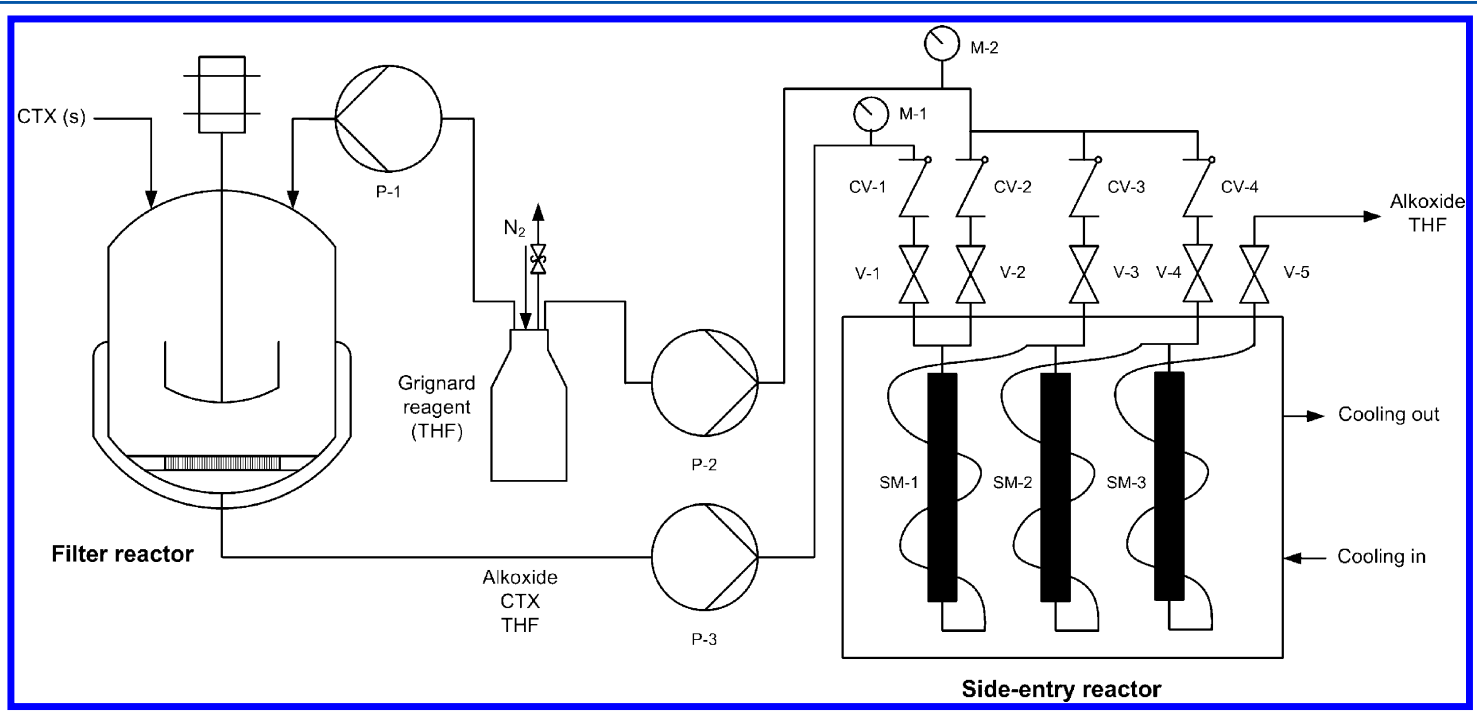

Figure 6. Setup used for the continuous alkylation of CTX with AllylMgCl (Grignard reagent). 
desired for each experiment (although the setup is designed for continuous operation if desired). An initial amount of THF was added to the filter reactor in order to allow mixing of the initial suspension. Next, typically about $50 \mathrm{~g}$ of CTX were added in order to create a suspension. Grignard reagent was then fed using a peristaltic pump (P-1), and the flow rate was regulated so that the reaction heat released could be removed by the cooling jacket. As the alkylation proceeded, the degree of CTX suspension (CTX excess above saturation) was decreased until a homogeneous solution was observed. Just before this point, another lot of CTX was added. Up to three CTX additions were made, until the desired amount of product was obtained. The Grignard reagent flow was stopped when an almost homogeneous solution was obtained, avoiding a high amount of CTX solids in suspension in order to facilitate filtering of the product, but keeping the solution saturated in CTX $(\sim 55 \mathrm{~g} / \mathrm{L})$. Further details about the operation of this reactor, focusing on continuous steady-state operation, can be found in references 38 and 39. The product from the filter reactor had a high concentration of dissolved alkoxide (between 100 and $300 \mathrm{~g} / \mathrm{L}$ ) in THF, and was saturated in CTX $(\sim 55 \mathrm{~g} / \mathrm{L})$. The concentration of alkoxide could be varied by changing the concentration of Grignard reagent (0.5-2 M). The filtered solution was then sent to a continuous side-entry reactor (three side entries) using a peristaltic pump (P-3). In this reactor, the CTX remains were partially reacted with additional Grignard reagent (peristaltic pump P-2). CV-1 to CV-4 in Figure 6 were check valves (Swagelok), V-1 to V-5 were standard valves (Swagelok), M-1 and M-2 were manometers used to detect potential plugging of the reactor, and SM-1 to SM-3 were 3/16 in., 27 elements stainless steel static mixers (Koflo). All tubing connections were made with $1 / 8$ in. stainless steel tube combined with $1 / 8$ in. PFA tubing where required (Swagelok). In this way, three solutions containing different concentrations of alkoxide and CTX were obtained (Table 1).

Table 1. Concentration of alkoxide and CTX in three different solutions obtained with the filter reactor coupled with the side-entry reactor ${ }^{a}$

\begin{tabular}{|lccc} 
& solution 1 & solution 2 & solution 3 \\
\hline alkoxide $(\mathrm{g} / \mathrm{L})$ & $136 \pm 6$ & $248 \pm 11$ & $284 \pm 9$ \\
CTX $(\mathrm{g} / \mathrm{L})$ & $23.6 \pm 0.9$ & $17.5 \pm 0.8$ & $3.7 \pm 0.1$
\end{tabular}

${ }^{a}$ The continuous hydrolysis of these solutions and subsequent separation of the organic and aqueous phases was tested individually.
The concentration of alkoxide was set around the expected value to be used for industrial operation, while a small concentration of CTX was left in the product from the side-entry reactor in order to observe any potential difficulties during hydrolysis and separation.

3.4.2. Continuous Hydrolysis of the Alkoxide Product. The continuous hydrolysis reaction was carried out by continuously pumping the alkoxide THF solution (covered with $\mathrm{N}_{2}$ ) and the acidic water to a $1 / 8 \mathrm{in}$. (3.2 mm) PFA Swagelok T-connection (Figure 7). The reaction mixture continued into a $3 \mathrm{~m}$ long $1 / 8$ in. PFA tube held in a water bath at room temperature to remove the heat of the reaction. Segmented flow with rapid mixing was observed. The reaction mixture had a white-cloudy to clear (transparent) yellow appearance for the organic phase and a partially cloudy aqueous phase, probably due to the presence of partially insoluble $\mathrm{Mg}$ salts.

3.5. Continuous L-L Separation of the Organic and Aqueous Phases after the Hydrolysis. 3.5.1. Preliminary Experiment to Compare Two Membrane Pore Sizes: 0.2 and $0.45 \mu \mathrm{m}$. A preliminary experiment was carried out to test the performance of two PTFE membranes with different pore sizes $(0.2$ and $0.45 \mu \mathrm{m})$. An organic solution containing THF and hydrolyzed allylcarbinol at a concentration of $430 \mathrm{~g} / \mathrm{L}$ and an aqueous solution containing acidic water and $\mathrm{MgCl}_{2}$ salts, obtained from a previous hydrolysis and phase separation by decantation, were mixed by means of a T-connection generating segmented flow, at a total flow rate ranging from 4 to $40 \mathrm{~mL} / \mathrm{min}$, keeping the flow ratio at $30 \%$ organic phase $/ 70 \%$ aqueous phase (this flow ratio was chosen because this was the organic and aqueous volume ratio obtained from the previous batch hydrolysis and decantation). This mixture was sent to the PTFE membrane separator, where the back-pressure regulator was set at 1 psi $(6.9 \mathrm{kPa})$. This was the resolution of the manometer used. The manometer was protected with a PFA gauge protector (Partek, Parker) which caused a slightly slower response of the manometer, especially at low pressures. If the organic phase was observed at the aqueous outlet, the back pressure was increased. The minimum pressure required to prevent the organic phase from exiting the device through the aqueous outlet was noted, and then the back-pressure was continuously increased until the aqueous phase was observed at the organic outlet. This was noted as the maximum pressure, thereby delimiting the operating window for this particular separation for the 2 membranes tested.

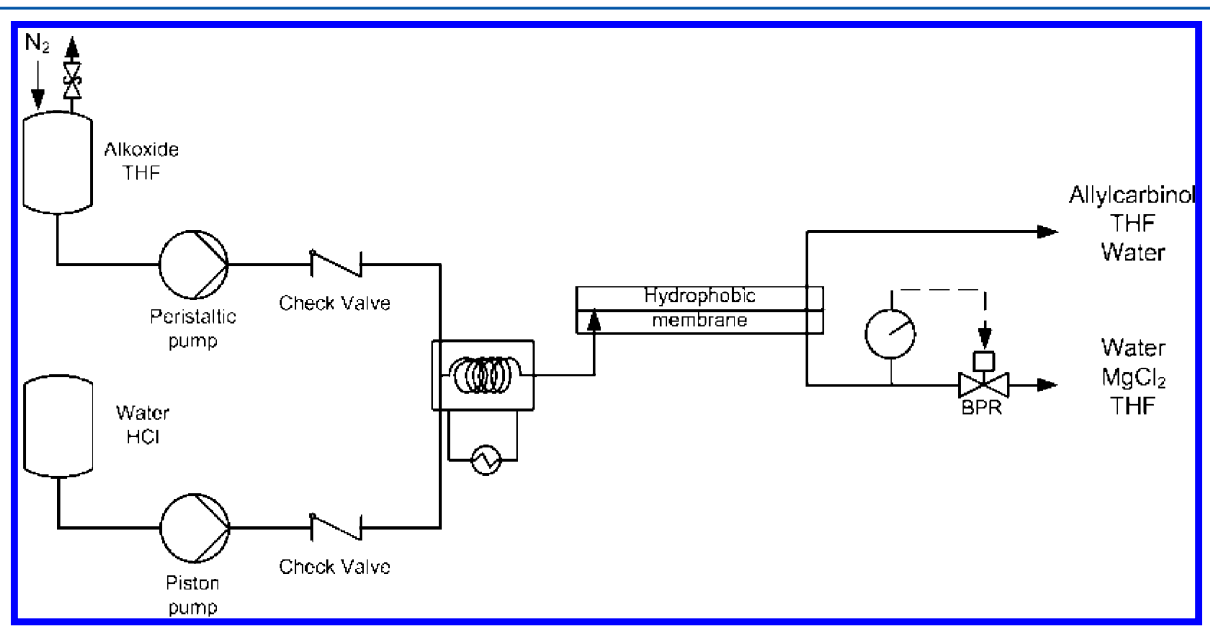

Figure 7. Setup for the continuous hydrolysis of an alkoxide THF solution and subsequent continuous separation of the resulting organic and aqueous solutions. 
3.5.2. Continuous Hydrolysis Coupled with Continuous $L-L$ Separation. The three solutions obtained at three different alkoxide concentrations (136, 248, and $284 \mathrm{~g} / \mathrm{L}$, Table 1) were individually hydrolyzed. The alkoxide THF solution and the acidic water were mixed at different flow rates and flow ratios. The hydrolysis product was collected before introducing it into the PTFE membrane separator in order to compare the membrane performance with a standard L-L separation by decantation in a glass tube. The hydrolysis product was also introduced into the membrane separator (equipped with a $0.45 \mu \mathrm{m}$ pore size membrane) and the back-pressure in the aqueous phase outlet was increased until the organic phase was observed in the organic phase outlet. However, the back-pressure was kept as low as possible in order to prevent water from crossing the PTFE membrane. The back-pressures used were noted, the organic and aqueous phase ratios in the two outlets were measured by a measuring flask in case the separation efficiency was not $100 \%$, and the organic and aqueous phases were analyzed via HPLC to quantify API intermediate and organic impurities concentration, and also via at-line NIR spectroscopy measurements to quantify the concentration of water in the organic stream and the concentration of THF in the aqueous stream.

3.6. Analytical Methods: HPLC and NIR. The concentrations of the API intermediate and the organic impurities in the aqueous and organic phases, as well as the alkoxide product used for the continuous hydrolysis were determined using HPLC analysis. A LaChrome Elite HPLC machine equipped with a Phenomenex Gemini C6-Phenyl column for reverse phase HPLC using a gradient method based on acetonitrile and aqueous buffer (ammonium formiate $\mathrm{pH}$ 9) as mobile phases was employed for the analysis of previously diluted samples. $50 \mu \mathrm{L}$ samples were diluted with $4.95 \mathrm{~mL}$ of THF. $50 \mu \mathrm{L}$ of this solution were further diluted with $950 \mu \mathrm{L}$ of mobile phase at time 0 of the gradient method (total dilution factor 2000). Samples were taken in triplicates, since repeatability of the dilution procedure was the major source of experimental error. Calibration curves were built from allylcarbinol and CTX standards, obtaining very satisfactory regression coefficients.

An at-line NIR spectroscopy analytical method was developed to measure THF concentration in water/aqueous phase (standard error of cross-validation SECV $=2.8 \mathrm{~g} / \mathrm{L}$ in the range $0-150 \mathrm{~g} / \mathrm{L}$ ) and water in a mixture of pure allylcarbinol $1.3 \mathrm{M}$ in THF, emulating the organic phase matrix (SECV = $0.55 \mathrm{~g} / \mathrm{L}$ in the range $0-36 \mathrm{~g} / \mathrm{L}$ ), using $1 \mathrm{~mL}$ disposable glass vials for a very fast and reliable measurement (for more details please consult the Supporting Information).

\section{RESULTS AND DISCUSSION}

4.1. Separation of Water and Toluene Using a Plate Coalescer. The separation of water and toluene was attempted at flow rates around $1-2 \mathrm{~mL} / \mathrm{min}$ with different flow ratios, since this value was considered significant to demonstrate reliability and ease of scalability. Perfect separation of both the toluene and water (no leakage of any of the phases into the other one) was achieved randomly and for very limited time periods. It was however easier to obtain a pure phase in one of the outlets, while the other outlet presented multiphase flow. This may be good enough for certain applications (e.g., partial phase separation for analytical purposes), but it was not considered useful for continuous L-L phase separation. Even though the back-pressures in the two outlets in prototype 2 were regulated to ensure continuous flow in the two outlets, operation was too cumbersome to achieve steady-state separation.
Prototype number 1 had a glass plate through which it was possible to observe the behavior of the two phases. The toluene phase was not forming a stable liquid layer on the PTFE surface. On the contrary, liquid slugs of the two phases had random contact with any of the two solid surfaces. It was difficult to evaluate whether there was any coalescence phenomenon taking place because the liquid slugs were too large already at the inlet of the device. The droplets were actually partially coalescing in the fluidic tubes before entering the device. Looking back to Figure 2, one can see that generating a $2 \mathrm{~cm}$ wide by $20 \mathrm{~cm}$ long interface would give an extremely low Laplace pressure, much lower than the flowdependent pressure gradient. It is much more energetically favorable to generate a $100 \mu \mathrm{m}$ high by $20 \mathrm{~cm}$ long interface, even if the aqueous phase has contact with the PTFE surface. This means that disregarding the coalescence effect, the two surfaces did not have any effect on the actual phase split. This depended uniquely on the capillary pressures given by the stainless steel outlet (aqueous stream) and the PTFE outlet (organic stream), whose inner diameters were $3 \mathrm{~mm}$ and $1.6 \mathrm{~mm}$ (inner diameter of the PFA tube which was inserted down to the fluoropolymer-coated surface in prototype 2), respectively. Assuming that the contact angle of water in toluene on PFA material was $160^{\circ}$ (it was probably lower) and on stainless steel $102^{\circ}$ (value as for Shelsol ${ }^{24}$ ), the difference in capillary pressure between the two outlets was only $75 \mathrm{~Pa}$. In order to have successful operation, the back pressure regulator in the aqueous outlet should be set at a value less than $75 \mathrm{~Pa}$ higher than the back pressure regulator in the organic outlet, so that the organic phase would preferentially take the organic outlet and the pressure gradient between the two phases would not be large enough to push the water through the PTFE outlet. However, this was obviously impossible taking into account the resolution of typical manometers and back-pressure regulators. Furthermore, even in the case that the water would not take the organic outlet, the organic phase would find two outlets with almost the same fluidic resistance, and thus the flow split could be almost $50 \%$ in each outlet. In conclusion, phase separation with a plate coalescer of these characteristics was found not feasible under realistic flow conditions.

4.2. Separation of Water and Toluene Using a PTFE Membrane Separator. The PTFE membrane separator allowed a perfect separation of the water and toluene streams at a flow rate up to $40 \mathrm{~mL} / \mathrm{min}$ for each phase (this was the maximum flow rate that could be obtained with the piston pumps) using a $0.45 \mu \mathrm{m}$ pore size membrane. Taking into account a typical value of $\gamma \cos \theta=0.005 \mathrm{~N} / \mathrm{m}$ (Kralj et al. ${ }^{4}$ ) and a typical pore radius around $0.225 \mu \mathrm{m}$, the capillary pressure given by this membrane should be around $45 \mathrm{kPa}$. Figure 8 shows the flow-dependent pressure drop given by the sum (eq 9) of (a) the pressure drop caused by water and toluene mixture in the rectangular channel on the first plate $(1 \mathrm{~mm}$ deep) before separation, (b) the pressure drop of the toluene phase through the membrane taking into account the $28 \mathrm{~cm}^{2}$ membrane-channel contact area, (c) the pressure drop of the toluene phase on the rectangular channel on the second plate ( $2 \mathrm{~mm}$ deep), and (d) the pressure drop of the toluene phase through a $1 \mathrm{~m}$ long $1 / 8 \mathrm{in}$. PFA tube (inner diameter $1.6 \mathrm{~mm}$ ) used as fluidic connection, assuming that a flow $Q$ containing $50 \%$ water and $50 \%$ toluene separates perfectly in the device (note that only the total pressure drop is depicted in Figure 8, not the individual components $\mathrm{a}-\mathrm{d}$ ). In order to evaluate the worst-case scenario, the viscosity of water has been taken for a flow $Q$ on channel 1 , and a toluene flow rate $Q / 2$ is assumed to 


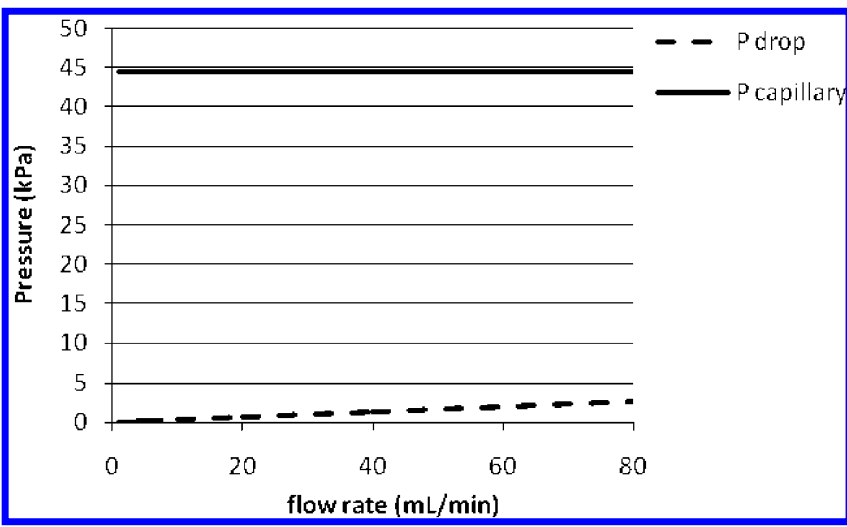

Figure 8. Flow-dependent pressure drop given by the sum (eq 9) of (a) the pressure drop caused by water and toluene mixture in the rectangular channel on the first plate, (b) the pressure drop of the toluene phase through the membrane taking into account the $28 \mathrm{~cm}^{2}$ membrane-channel contact area, (c) the pressure drop of the toluene phase on the rectangular channel on the second plate, and (d) the pressure drop of the toluene phase through a $1 \mathrm{~m}$ long $1 / 8$ in. PFA tube (inner diameter $1.6 \mathrm{~mm}$ ) used as fluidic connection, assuming that a flow $Q$ containing 50\% water and 50\% toluene separates perfectly in the device.

cross the membrane using all the area available. Using information from the manufacturer, the number of pores of this membrane has been calculated to be around $5.2 \times 10^{8}$ pores/ $\mathrm{cm}^{2}$, and therefore the total number of pores available should be around $1.46 \times 10^{10}$. The operating window is the area between the upper limit of the figure ( $P$ capillary) and the lower limit $(P$ drop $)$. If a back-pressure regulator is used in the aqueous outlet to generate a fluidic resistance several times higher than the lower limit of the operating window but below the capillary pressure, excellent separation will be obtained. It would thus be possible to separate water and toluene mixtures at much higher flow rates, since the operating window is very large.

$$
\begin{aligned}
\Delta P_{\text {inlet }}= & \Delta P_{\text {rec.channel 1 }}^{\text {water }+ \text { toluene, } Q}+\Delta P_{\text {membrane }}^{\text {toluene, } Q / 2} \\
& +\Delta P_{\text {rec.channel } 2}^{\text {toluene, } Q / 2}+\Delta P_{\text {tube }}^{\text {toluene, } Q / 2}
\end{aligned}
$$

4.3. Continuous Hydrolysis and L-L Phase Separation of an API Intermediate Dissolved in THF. Just as density difference is the driving force for decantation and centrifugal separation, surface tension is the driving force for capillary L-L separation. The separation of the organic and aqueous mixture obtained from the hydrolysis of the alkoxide product is challenging for both separation strategies. The density of the two phases is almost the same, differing by only $25 \mathrm{~kg} / \mathrm{m}^{3}$ approximately. This is due to the fact that THF dissolves a high amount of high molecular weight organic products (principally the API intermediate) and a small amount of water $(\sim 20-40 \mathrm{~g} / \mathrm{L})$, and the aqueous phase contains some $\mathrm{MgCl}_{2}$ salts and a considerable amount of THF (200-300 g/L). The surface tension of THF in air is $26.4 \mathrm{mN} / \mathrm{m}$ at $20{ }^{\circ} \mathrm{C}$ (similar to that of toluene, $28.4 \mathrm{mN} / \mathrm{m}$ ), while that of water in air is $72.8 \mathrm{mN} / \mathrm{m}$ for the same temperature. However, the surface tension at the aqueous and organic interface is not as high as the water-toluene surface tension $(36 \mathrm{mN} / \mathrm{m})$, and consequently the separation by means of a PTFE membrane is much more challenging.

4.3.1. Preliminary Experiment to Compare Two Membrane Pore Sizes: 0.2 and $0.45 \mu \mathrm{m}$. A preliminary experiment was carried out to test the performance of two PTFE membranes with different pore sizes $(0.2$ and $0.45 \mu \mathrm{m})$. The operating window for the two membranes is shown in Figure 9.

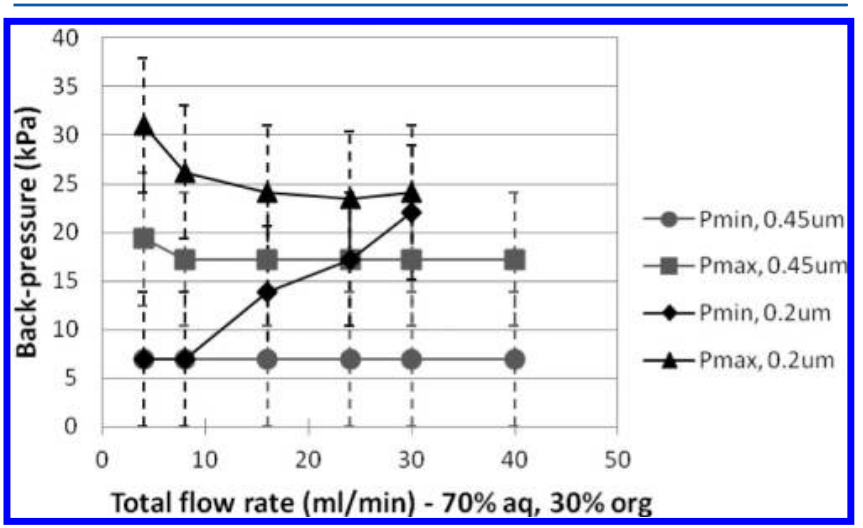

Figure 9. Operating windows of the 0.2 and $0.45 \mu \mathrm{m}$ membranes for the separation of an organic ( $430 \mathrm{~g} / \mathrm{L}$ of allylcarbinol in THF) and aqueous mixture (water and $\mathrm{MgCl}_{2}$ salts), pumped at a flow ratio $70 \%$ aqueous phase $/ 30 \%$ organic phase.

It is remarkable that the maximum operating pressure for the $0.2 \mu \mathrm{m}$ pore size membrane showed a decreasing trend with increasing flow rate, even though the maximum operating pressure should depend only on the capillary pressure and should thus be independent of the flow rate (as observed with the $0.45 \mu \mathrm{m}$ membrane). However, the resolution of the manometer used (equipped with a PFA protector causing a slightly delayed response) was $1 \mathrm{psi}(6.9 \mathrm{kPa})$, and therefore the observed variations in these curves may be due to a biased reading. It is speculated that the variable maximum operating pressure of the $0.2 \mu \mathrm{m}$ membrane could also be due to slight defects caused by bending of the membrane at high pressure, since-as explained in the Materials and Methods section-it was finally chosen not to use a membrane support. Bending could result in enlargement of weak pores, consequently decreasing the effective capillary pressure. This effect is perhaps more noticeable at high flow rates. Future work should therefore take into account the possibly deleterious effects of membrane bending at high pressure. Nevertheless, these observations did not apply to the $0.45 \mu \mathrm{m}$ membrane and therefore experiments were continued with this membrane.

While the $0.45 \mu \mathrm{m}$ membrane could be used with perfect phase separation up to the maximum flow rate tested $(40 \mathrm{~mL} / \mathrm{min})$, the $0.2 \mu \mathrm{m}$ membrane required a higher back-pressure to push all the organic phase through it. At $30 \mathrm{~mL} / \mathrm{min}$ and above the pressure drop given by the membrane became closer to the capillary pressure and a small amount of aqueous phase was observed in the organic phase outlet. Even though THF and toluene have similar surface tensions with air, the maximum back-pressure for the $0.45 \mu \mathrm{m}$ membrane $(15-20 \mathrm{kPa})$ is significantly lower than the capillary pressure for the system water-toluene $(45 \mathrm{kPa})$, due to the partial miscibility of THF in the aqueous phase (100$150 \mathrm{~g} / \mathrm{L}$ measured from previous experiments).

It is surprising that the maximum back-pressure for the $0.2 \mu \mathrm{m}$ membrane is closer to the one for the $0.45 \mu \mathrm{m}$ membrane than expected. Note that using eq 6 applied to round capillaries, the expected ratio of capillary pressures between the $0.2 \mu \mathrm{m}$ membrane and the $0.45 \mu \mathrm{m}$ membrane should be $0.45 / 0.2$, i.e., 2.25 , while the value obtained experimentally is $\sim 25 / 17=1.5$ (Figure 9). Disregarding the resolution of the manometer used, this is most probably due to a nonuniform 
pore size distribution. The commercial catalogue of PTFE membranes provided by Sartorius reports a bubble point measurement of 0.8 and 1 bar for the 0.45 and $0.2 \mu \mathrm{m}$ membranes, respectively, in both cases evaluated with isopropanol according to the standard DIN 58355. The bubble point measurement provides an indication of the maximum pore size for each membrane. The ratio of bubble point measurements is 1.25 , which corresponds rather well to the ratio of experimental maximum pressures (1.5). Since the maximum pressures reported were noted as soon as a drop of aqueous phase was detected in the organic phase outlet, these measurements correlate-just as the bubble point measurements - to the maximum pore size of each membrane. Therefore, even though in the design phase the nominal pore size value was used for calculation of the expected capillary pressure, one must consider that each membrane has a certain pore size distribution, and in this case it was observed that the membrane with the smallest pores had an apparent larger pore size distribution than the membrane with the larger pores, with the result of similar maximum allowed pressures. Hence, in the design phase, one should consider measuring the effective maximum pore size experimentally, since this value will determine when an aqueous 'leak' will be found in the organic phase outlet. This test will also be useful to confirm membrane integrity.

Taking into account the larger area of the $0.45 \mu \mathrm{m}$ membrane operating window, it was decided to proceed with the experiments using only this membrane pore size. The $0.2 \mu \mathrm{m}$ membrane could only be used if the membrane area was enlarged, requiring a larger setup, and could perhaps be more sensitive to fouling problems, thus presenting no obvious advantages for this particular application.

4.3.2. Continuous Hydrolysis Coupled with Continuous $L-L$ Separation. The three alkoxide solutions produced in continuous mode with the filter reactor coupled with the sideentry reactor, and containing different concentrations of alkoxide and CTX as detailed in Table 1, were used for continuous hydrolysis and L-L phase separation. The range of alkoxide concentrations (100-300 g/L) has been set around the set-point expected for industrial application. The three alkoxide solutions were reacted with water containing sufficient $\mathrm{HCl}$ to convert all the magnesium salts into the soluble form $\left(\mathrm{MgCl}_{2}\right)$, thereby dropping the $\mathrm{pH}$ to $1-2$. The hydrolysis products were separated both by decantation and membrane separation. While the separations by decantation sometimes gave cloudy organic and aqueous phases, the organic phase from the membrane separation was always clear, and the aqueous phase looked only slightly cloudy. The apparent 'clearing' of the organic and aqueous phases could be due to a reduction of the degree of emulsion of both phases in the membrane separator that by having a channel with a stainless steel surface on one side (body), and a PTFE surface on the other side (membrane) could work as a plate coalescer. ${ }^{24}$ Furthermore, the PTFE membrane allows a more efficient and faster separation than the decantation process, resulting in less emulsion formation. Clearing of the phases could also be an indication of potential fouling of the membrane due to accumulation of nonsoluble species at the interface. Solid deposition was actually found inside the separator when it was dismantled, particularly in specific areas (dead zones). Nevertheless, during the relatively short-term experiments carried out, the performance of the PTFE membrane separator did not decrease with time. Yet, as further discussed below, long-term experiments should be performed in order to identify any fouling issues.
The continuous phase separation of the three solutions listed in Table 1 was successful with excellent separation (100\% of the allylcarbinol in the organic phase). However, among the flow ratios tested (Table 2), solutions 2 and 3 (highest alkoxide

Table 2. Flow ratios of alkoxide solution and acidic water tested for solutions 1, 2, and 3, indicating the measured flow out of the separator (organic phase and aqueous phase out) and the back-pressure setting ${ }^{a}$

\begin{tabular}{|c|c|c|c|c|c|}
\hline & $\begin{array}{c}\text { alkoxide } \\
(\mathrm{mL} / \mathrm{min})\end{array}$ & $\begin{array}{l}\text { ac. water } \\
(\mathrm{mL} / \mathrm{min})\end{array}$ & $\begin{array}{c}\text { org. out } \\
(\mathrm{mL} / \mathrm{min})\end{array}$ & $\begin{array}{l}\text { aq. out } \\
(\mathrm{mL} / \mathrm{min})\end{array}$ & $\begin{array}{c}\text { back- } \\
\text { pressure } \\
(\mathrm{kPa})\end{array}$ \\
\hline \multirow[t]{5}{*}{ solution 1} & 4.0 & 4.0 & 3.0 & 5.3 & 0 \\
\hline & 6.0 & 6.0 & 4.2 & 7.6 & 0 \\
\hline & 4.0 & 8.0 & 1.9 & 10.1 & 0 \\
\hline & 4.0 & 4.8 & 2.7 & 7.5 & 0 \\
\hline & 4.0 & 5.6 & 2.2 & 7.9 & 0 \\
\hline \multirow[t]{3}{*}{ solution 2} & 2.0 & 2.0 & - & - & - \\
\hline & 4.0 & 4.0 & - & - & - \\
\hline & 2.0 & 4.0 & 0.8 & 5.0 & 7 \\
\hline \multirow[t]{5}{*}{ solution 3} & 2.0 & 4.0 & 1.2 & 4.7 & 9 \\
\hline & 4.0 & 4.0 & - & - & - \\
\hline & 4.0 & 2.0 & - & - & - \\
\hline & 2.0 & 6.0 & - & - & - \\
\hline & 4.0 & 4.8 & - & - & - \\
\hline
\end{tabular}

${ }^{a}$ Missing values in the table indicate that excellent separation (no cross-contamination) could not be achieved for the back-pressures tested. Lack of volume balance for some of the tests is attributed to experimental errors.

concentration) gave only successful separations when the ratio alkoxide solution to water was 1:2. In contrast, solution 1 (lowest alkoxide concentration) gave excellent separation for all the flow ratios tested. This is thought to be related to solubility of the salts and not to the performance of the membrane separator, since the separation by decantation was also cumbersome, showing a high degree of emulsion and cloudy phases. Even though the solubility of $\mathrm{MgCl}_{2}$ salts in water is very high (around $500 \mathrm{~g} / \mathrm{L}$, that is $\sim 5 \mathrm{M})$-compared to the molar concentration of alkoxide product, $0.5-1 \mathrm{M}$-the aqueous phase had a cloudy appearance, which may be due to the high concentration of THF, lowering $\mathrm{Mg}$ salts solubility (see Figure $10 \mathrm{~b}$ ).

Figure 10 compares the allylcarbinol and CTX concentrations in the organic and aqueous phases obtained by decantation and by continuous membrane separation, as measured by HPLC (a), and the concentration of THF in the aqueous phase and the concentration of water in the organic phase, measured by at-line NIR spectroscopy measurements (b). Figure 10b shows that the aqueous phase contains a considerable amount of dissolved THF (150-250 g/L), while the organic phase contains a small amount of water $(17-45 \mathrm{~g} / \mathrm{L})$. The water concentration in the organic phase for solutions 2 and 3 (highest alkoxide concentration) obtained by decantation could not be measured because the organic phase was very cloudy and caused too much scattering for the NIR transmission measurements (the samples were difficult to filter with common syringe filters due to the solvent strength of THF). The transfer of THF from the alkoxide solution to the aqueous phase entails an increase in concentration of the allylcarbinol solution compared to the original concentration (Table 1).

The concentrations for solution 2 are difficult to compare because the flow ratios used in decantation and membrane separation were different. However, one can see that even though 


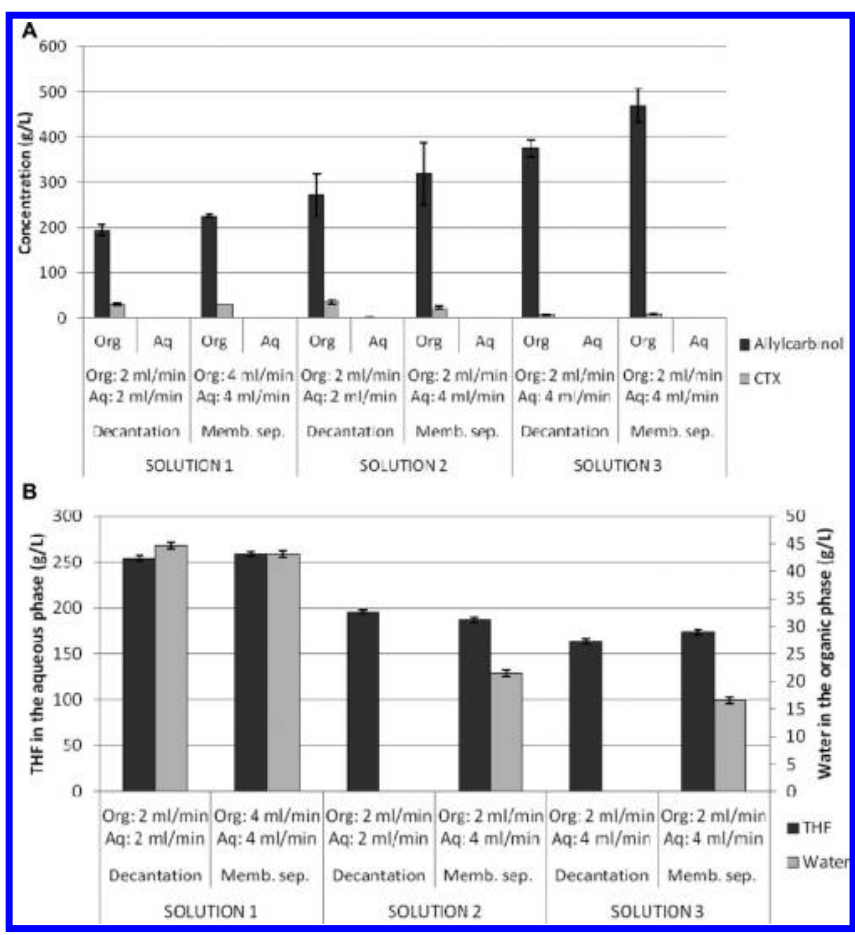

Figure 10. (A) Allylcarbinol and CTX concentrations in the organic and aqueous phases obtained by decantation and by continuous membrane separation, for solutions 1-3. (B) Concentration of THF in the aqueous phase and water in the organic phase, after decantation and membrane separation, for solutions $1-3$. The water concentration in the organic phase for solutions 2 and 3 obtained by decantation could not be measured because the organic phase was very cloudy and caused too much scattering for the NIR transmission measurements.

the double amount of water was used in the membrane separation compared to the decantation, the THF concentration in the aqueous phase decreased very little. This is thought to be due to a dilution of the $\mathrm{Mg}$ salts in the aqueous phase, decreasing the salting-out effect and thus allowing more THF in solution. The result is an up-concentration of allylcarbinol in the organic phase (Figure 10a), which could otherwise seem surprising.

Solutions 1 and 3 also show an increase in the allylcarbinol concentration by using the membrane separation, which may be due to a slight increase in the THF transfer to the aqueous phase (Figure 10b). It is probably also related to a decrease in the water concentration in the organic phase, as shown for solution 1 . Unfortunately, as already mentioned, the water concentrations for the decantation from solutions 2 and 3 could not be measured. It is difficult to find a reason for the slight differences between decantation and membrane-based separation, and perhaps they are within experimental error. The observed differences could be due to fine emulsions present in the samples obtained by decantation and nonperfect separation due to the experimental procedure inherent to the decantation process. The membranebased separation removes emulsion limitations to a high degree.

Figure 11 shows the effect of the flow ratio on the concentration of allylcarbinol, CTX, THF in the aqueous phase, and water in the organic phase, for solution 1. As discussed above, even if a larger amount of water is used for the hydrolysis, the resulting API intermediate concentration in the organic phase increases, due to a reduction of the salting-out effect in the aqueous phase allowing more THF to be dissolved.

It is well noted that this particular L-L phase separation produces an aqueous waste stream containing relatively large

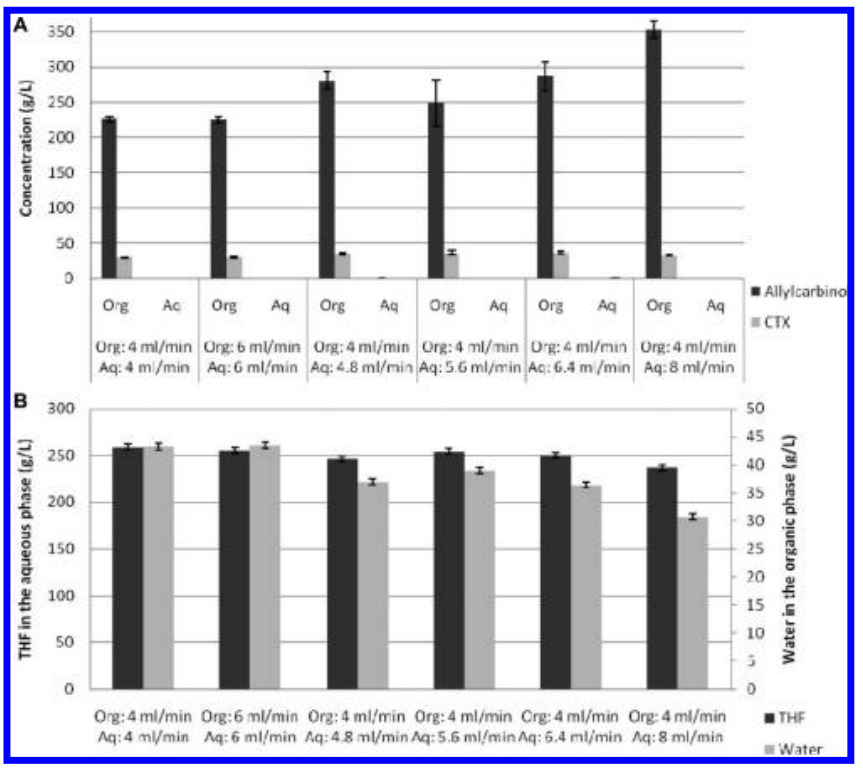

Figure 11. (A) Effect of the flow ratio between alkoxide solution and acidic water on the allylcarbinol and CTX concentration in the organic and aqueous phases obtained by membrane separation. (B) Effect of the flow ratio between alkoxide solution and acidic water on the THF concentration in the aqueous phase and the water concentration in the organic phase obtained by membrane separation.

amounts of THF. The organic phase contains a small amount of water as well, which could cause problems downstream. These two concentrations can be decreased by processing alkoxide solutions at higher concentrations, as expected from the application of the Setschenow equation, ${ }^{41}$ and as clearly shown in Figure 10. However, using alkoxide solutions at high concentration may cause problems upstream as well, and the separation is less flexible in terms of flow ratio (Table 2). An obvious solution to avoid the "loss" of solvent in the aqueous stream while decreasing the water concentration in the organic stream would be using a different solvent with lower miscibility with water-such as methyl-THF or CPME ${ }^{42}$ - bringing about a concomitant easier phase separation as well by an increase of the surface tension.

4.4. Discussion on Operability and Scalability. A PTFE membrane L-L separator capable of achieving excellent separation of organic and aqueous phases at moderate to high flow rates $(0-80 \mathrm{~mL} / \mathrm{min}$ for toluene-water and $0-40 \mathrm{~mL} / \mathrm{min}$ for a mixture of partially miscible aqueous and THF phases, demonstrating potential to achieve higher flow rates) has been presented. The separation setup demonstrated flexibility in a large operating window both in terms of flow rate and organic/ aqueous flow ratio by simply regulating the back-pressure. Such flexibility is important in view of practical and robust operability of the system. The hydrolysis and separation of solutions 2 and 3 (highest alkoxide concentrations) was only successful when the flow ratio of water to alkoxide solution was 2:1 (Table 2). However, separation difficulties were attributed to solubility limitations of $\mathrm{Mg}$ salts, probably causing changes in the surface tension which are difficult to predict. Nothing indicatesaccording to the physical analysis of the separation processthat the separation should be affected by the flow ratio in a system with homogeneous solutions with a well-defined surface tension.

The active membrane area applied in this study is only $28 \mathrm{~cm}^{2}$, and the aqueous and organic channels have volumes 
of 2.8 and $5.6 \mathrm{~mL}$, respectively. The separation device thus has lab bench dimensions; despite that, it can process flows similar to those expected for industrial-scale production $(\sim 100 \mathrm{~mL} /$ $\left.\min ^{33,34}\right)$. The separator itself is relatively simple to fabricate and thus easy to scale-up (enlarge) or scale-out (replicate). Possible scaling-up/-out strategies would be: (1) using a large coiled membrane as, for example, that used in reverse osmosis; (2) employ a hollow fiber setup; or (3) simply install several plates in parallel, sandwiching membranes in between, as used in PEM fuel cells. The last strategy would allow for a high flexibility in terms of throughput, since membrane panels could be added or removed as required.

Fouling or blockages occurring on the aqueous side could possibly be removed by reversing the flow in the system, which should be feasible since the separator can be constructed symmetrically. The device could also work as a stand-alone unit with automatic regulation by, for example, measuring the capacitance in the outlets, ${ }^{16}$ which as demonstrated by Mendorf et al. ${ }^{16}$ can differentiate aqueous/organic streams and detect when segmented flow is occurring. The control strategy could, for example, be starting the unit and smoothly increasing the backpressure until segmented flow disappears in the aqueous phase outlet (detecting only aqueous phase), and organic phase is detected in the organic phase outlet. An alarm could be set up for the operators to check the system when the back-pressure approaches the capillary pressure or when segmented flow is detected in the organic phase stream, indicating that the flow must be reversed to eliminate fouling or blockage, or eventually one or more of the membranes need to be cleaned or replaced. Naturally, future studies should also focus on the long-term performance of these membranes.

The key advantages of membrane-based L-L separation compared to other separation methods relying on surface tension are the small pore sizes (providing a high capillary pressure) and the large number of pores per unit area (causing a low pressure drop), all in all offering a large operating window in terms of flow rate and flow ratio. It has also been shown that the channels carrying the liquids offer a low pressure drop, decoupling the channel size and the interface size which limits operability in other designs. The large number of pores and the small pore sizes are standard in PTFE membranes, and thus the separator is easy to fabricate and assemble, compared to other designs where several microchannels or micropillars need to be microfabricated. $^{29,30}$ In conclusion, membrane-based L-L separation seems the most robust of the surface tension-based separation methods which have been reviewed. The only limitation seems to be the situation where the organic phase carries solids in suspension that should remain in the organic phase but are larger than the membrane pores. ${ }^{29}$ However, the latter problem could perhaps be solved by using a hydrophilic membrane prewetted with aqueous phase allowing only aqueous phase to cross. Unfortunately, no work or discussion has been found in the literature describing such an application or studying its feasibility. Note that this may not be a simple material-selection task, since many polymers do not have a chemical compatibility as broad as that of PTFE. Nevertheless, hydrophilic PTFE membranes are also available commercially.

The presented membrane-separation setup is designed to perform only a L-L separation operation, while mixing and extraction occurs in a different mixing unit, in co-current mode. Therefore, one mixing and separation cycle corresponds to one equilibrium stage. ${ }^{4}$ It would be interesting to develop multistage countercurrent L-L extraction using membranes, as used in analytical chemistry in the form of MMLLE. ${ }^{1,3}$ The limitation would then become the diffusion rate through the membrane, which could be a subject for future investigation.

Regarding industrial acceptance, membrane-based L-L separation must demonstrate robustness, flexibility and simple and cost-effective operation compared to L-L extraction/ separation using hydrocyclones. ${ }^{18-21}$ One of the advantages of membrane-based L-L separation as shown in this work is that it both scales up and down relatively easy. It is very efficient when separating liquids with a high surface tension, such as organic and aqueous solutions. This type of separation problem is rather common in the pharmaceutical industry. L-L extraction operations involving different organic solvents may be difficult to carry out using membranes, due to the low surface tensions that are to be expected. Hydrocyclones are on the contrary very efficient in separating liquids as long as they have a minimal density difference. All in all, a generic methodology would be useful in order to identify the most appropriate operation for a given separation task, which requires more experimental studies and the development of surface tension prediction tools for complex solvent-solute mixtures.

\section{CONCLUSIONS}

Microscale L-L separation based on surface tension has briefly been reviewed and physically analyzed, concluding that hydrophobic membrane L-L separation is one of the most flexible and robust separation methods. Two separation devices, a plate coalescer and a membrane separator, were constructed and tested with a water and toluene separation. While the plate coalescer showed very small flexibility due to the very low Laplace pressure enabling the separation, the membrane separator demonstrated operability in a large operating window, thanks to decoupling the flow-dependent pressure drop gradient between the phases which disrupts the interface and the Laplace pressure which preserves it. This can be achieved by constructing relatively large microchannels to drive the fluids with negligible pressure drop, using a membrane with a large number of pores per unit area and offering high capillary pressure, and installing a back-pressure regulator in the aqueous phase outlet to force the organic phase through the membrane. The result is a perfect separation (no cross-contamination) independent of flow rate and flow ratio within a large operating window.

Water-toluene mixtures at flow rates up to $40 \mathrm{~mL} / \mathrm{min}$ per phase were perfectly separated with only $28 \mathrm{~cm}^{2}$ of active membrane area, and a physical analysis of the separation shows that higher flow rates could easily be obtained. The hydrolysis of an alkoxide solution (an API intermediate dissolved in THF) with water and $\mathrm{HCl}$ was carried out in continuous mode. The organic and aqueous solutions (containing partially soluble $\mathrm{Mg}$ salts) were continuously separated using the membrane separator. Total flow rates up to $40 \mathrm{~mL} / \mathrm{min}$ were tested at different flow ratios between the alkoxide and the acidic water solution. Even though water and THF were partially miscible and thus kept a low surface tension, perfect separation of the phases could be obtained. The membrane L-L separator's simple construction and assembly suggest that it should be relatively simple to scale-up/out, thereby becoming a flexible module for continuous pharmaceutical manufacturing.

\section{ASSOCIATED CONTENT}

\section{S Supporting Information}

Development of NIR spectroscopy analytical method for at-line $\mathrm{THF}$ and water measurement in aqueous and organic phase, 
respectively. This material is available free of charge via the Internet at http://pubs.acs.org.

\section{AUTHOR INFORMATION}

\section{Corresponding Author}

*Telephone: +45 45252970. Fax: +45 45932906. E-mail: kvg@ kt.dtu.dk.

\section{Notes}

The authors declare no competing financial interest.

\section{ACKNOWLEDGMENTS}

We thank the Technical University of Denmark and Lundbeck A/S for technical and financial support, and Michael Lindaa, Ivan Pedersen and Mette Larsen for technical assistance in the construction of the phase separators. The authors also thank Accoat A/S for coating the plate coalescer, Sartorius Stedim for lending the PTFE membrane filters and Q-Interline A/S for support with the NIR spectrophotometer.

\section{REFERENCES}

(1) Nord, L.; Karlberg, B. Anal. Chim. Acta 1980, 118, 285.

(2) Jönsson, J. Å.; Mathiasson, L. I. Chromatogr. A 2000, 902, 205.

(3) Cai, Z.; Fang, Q.; Chen, H.; Fang, Z. Anal. Chim. Acta 2006, $556,151$.

(4) Kralj, J. G.; Sahoo, H. R.; Jensen, K. F. Lab Chip 2007, 7, 256.

(5) Hartman, R. L.; Jensen, K. F. Lab Chip 2009, 9, 2495.

(6) Jensen, K. F. Chem. Eng. Sci. 2001, 56, 293.

(7) Behr, A.; Brehme, V. A.; Ewers, C. L. J.; Grön, H.; Kimmel, T.; Küppers, S.; Symietz, I. Eng. Life Sci. 2004, 4, 15.

(8) LaPorte, T. L.; Wang, C. Curr. Opin. Drug Discovery Dev. 2007, 10, 738 .

(9) Plumb, K. Chem. Eng. Res. Des. 2005, 83, 730.

(10) Pollet, P.; Cope, E. D.; Kassner, M. K.; Charney, R.; Terett, S. H.; Richman, K. W.; Dubay, W.; Stringer, J.; Eckert, C. A.; Liotta, C. L. Ind. Eng. Chem. Res. 2009, 48, 7032.

(11) International Conference on Harmonisation (ICH), QbD Guidelines (Q8, Q9 and Q10). (a) ICH Q8 Pharmaceutical Development, (R2); U.S. Department of Health and Human Services, Food and Drug Administration, Center for Drug Evaluation and Research (CDER): Rockville, MD, Aug 2009. (b) ICH Q9 Quality Risk Management; U.S. Department of Health and Human Services, Food and Drug Administration, Center for Drug Evaluation and Research (CDER): Rockville, MD, June 2006. (c) ICH Q10 Pharmaceutical Quality System; U.S. Department of Health and Human Services, Food and Drug Administration, Center for Drug Evaluation and Research (CDER): Rockville, MD, April 2009.

(12) Food and Drug Administration (FDA), US, 2004. PAT guidance for industry.

(13) Sahoo, H. R.; Kralj, J. G.; Jensen, K. F. Angew. Chem. 2007, 119, 5806.

(14) Hartman, R. L.; Naber, J. R.; Buchwald, S. L.; Jensen, K. F. Anoew. Chem. Int. Ed. 2010, 49, 899.

(15) Hartman, R. L.; Naber, J. R.; Zaborenko, N.; Buchwald, S. L.; Jensen, K. F. Org. Process Res. Dev. 2010, 14, 1347.

(16) Mendorf, M.; Nachtrodt, H.; Mescher, A.; Ghaini, A.; Agar, D. W. Ind. Eng. Chem. Res. 2010, 49, 10908.

(17) Aota, A.; Nonaka, M.; Hibara, A.; Kitamori, T. Angew. Chem. 2007, 119, 896.

(18) Kraai, G. N.; van Zwol, F.; Schuur, B.; Heeres, H. J.; de Vries, J. G. Angew. Chem. Int. Ed. 2008, 47, 3905.

(19) Kraai, G. N.; Schuur, B.; van Zwol, F.; van de Bovenkamp, H. H.; Heeres, H. J. Chem. Eng. I. 2009, 154, 384.

(20) Schuur, B.; Hallett, A. J.; Winkelman, J. G. M.; de Vries, J. G.; Heeres, H. I. Org. Process Res. Dev. 2009, 13, 911.

(21) Schuur, B.; Winkelman, J. G. M.; de Vries, J. G.; Heeres, H. J. Chem. Eng. Sci. 2010, 65, 4682.
(22) Okubo, Y.; Maki, T.; Aoki, N.; Hong Khoo, T.; Ohmukai, Y.; Mae, K. Chem. Eng. Sci. 2008, 63, 4070.

(23) Okubo, Y.; Toma, M.; Ueda, H.; Maki, T.; Mae, K. Chem. Eng. J. 2004, 101, 39.

(24) Kolehmainen, E.; Turunen, I. Chem. Eng. Process 2007, 46, 834.

(25) Chen, X.; Lu, H.; Jiang, W.; Chu, L.; Liang, B. Ind. Eng. Chem. Res. 2010, 49, 9279.

(26) Zhao, B.; Moore, J. S.; Beebe, D. I. Science 2001, 291, 1023.

(27) Smirnova, A.; Shimura, K.; Hibara, A.; Proskurnin, M. A.; Kitamori, T. Anal. Sci. 2007, 23, 103.

(28) Varnik, F.; Truman, P.; Wu, B.; Uhlmann, P.; Raabe, D.; Stamm, M. Phvs. Fluids 2008, 20, 072104.

(29) Castell, O. K.; Allender, C. J.; Barrow, D. A. Lab Chip 2009, 9, 388 .

(30) Berthier, J.; Tran, V.; Mittler, F.; Sarrut, N. Sens. Actuators, A 2009, 149, 56.

(31) Aota, A.; Mawatari, K.; Takahashi, S.; Matsumoto, T.; Kanda, K.; Anraku, R.; Hibara, A.; Tokeshi, M.; Kitamori, T. Microchim. Acta 2009, 164, 249.

(32) Aota, A.; Mawatari, K.; Kitamori, T. Lab Chip 2009, 9, 2470.

(33) Barthe, P.; Guermeur, C.; Lobet, O.; Moreno, M.; Woehl, P.; Roberge, D. M.; Bieler, N.; Zimmermann, B. Chem. Eng. Technol. 2008, 31, 1146.

(34) Roberge, D. M.; Bieler, N.; Mathier, M.; Eyholzer, M.; Zimmermann, B.; Barthe, P.; Guermeur, C.; Lobet, O.; Moreno, M.; Woehl, P. Chem. Eng. Technol. 2008, 31, 1155.

(35) Kockmann, N.; Roberge, D. M. Chem. Eng. Technol. 2009, 32, 1682.

(36) Silverman, G. S.; Rakita, P. E. Handbook of Grignard Reagents; CRC Press: New York, 1996.

(37) Roberge, D. M.; Ducry, L.; Bieler, N.; Cretton, P.; Zimmermann, B. Chem. Eng. Technol. 2005, 28, 318.

(38) Cervera-Padrell, A. E.; Nielsen, J. P.; Jønch Pedersen, M.; Müller Christensen, K.; Mortensen, A. R.; Skovby, T.; Dam-Johansen, K.; Kiil, S.; Gernaey, K. V. Org. Process Res. Dev. 2012, DOI: 10.1021/ op2002563.

(39) Müller Christensen, K.; Jønch Pedersen, M.; Dam-Johansen, K.; Lønberg Holm, T.; Skovby, T.; Kiil, S. Chem. Eno. Sci. 2012, 71, 111.

(40) Bruus, H. Theoretical Microfluidics; Oxford University Press: Oxford, UK, 2008.

(41) Ni, N.; Yalkowsky, S. H. Int. I. Pharm. 2003, 254, 167.

(42) Watanabe, K.; Yamagiwa, N.; Torisawa, Y. Org. Process Res. Dev. 2007, 11, 251. 